\title{
Disabling the Nuclear Translocalization of RelA/ NF- $\kappa B$ by a Small Molecule Inhibits Triple-Negative Breast Cancer Growth
}

\author{
Hirotaka Kanzaki' \\ Avradip Chatterjee (D) \\ Hanieh Hossein Nejad \\ Ariani (ID) ${ }^{\prime}$ \\ Xinfeng Zhang ${ }^{2}$ \\ Stacey Chung ${ }^{2}$ \\ Nan Deng ${ }^{3,4}$ \\ V Krishnan Ramanujan ${ }^{4}$ \\ Xiaojiang Cui ${ }^{1,2,4}$ \\ Mark I Greene ${ }^{5}$ \\ Ramachandran Murali ${ }^{1}$ \\ 'Department of Biomedical Sciences, \\ Research Division of Immunology, \\ ${ }^{2}$ Department of Surgery, ${ }^{3}$ Biostatistics \\ and Bioinformatics Research Center, \\ ${ }^{4}$ Samuel Oschin Comprehensive Cancer \\ Institute, Cedars-Sinai Medical Center, \\ Los Angeles, CA, 90048, USA; \\ ${ }^{5}$ Department of Pathology and \\ Laboratory of Medicine, University of \\ Pennsylvania, Philadelphia, PA, \\ 19104, USA
}

Introduction: Constitutive activation of $\mathrm{NF}-\mathrm{\kappa B}$ has been implicated as being contributive to cancer cell growth, drug resistance, and tumor recurrence in many cancers including breast cancer. Activation of NF- $\kappa \mathrm{B}$ leads to nuclear translocation of RelA, a critical component of the NF- $\mathrm{KB}$ transcription factor complex, which subsequently binds to specific DNA sites and activates a multitude of genes involved in diverse cell functions. Studies show that triplenegative breast cancer (TNBC) cells possess constitutively active NF- $\kappa \mathrm{B}$ and concomitantly have higher levels of nuclear localization of RelA than cytoplasmic RelA. This feature is considered to be associated with the response to chemotherapy. However, currently, there is no specific inhibitor to block nuclear translocation of RelA.

Methods: A structure-based approach was used to develop a small-molecule inhibitor of RelA nuclear translocation. The interaction between this molecule and RelA was verified biophysically through isothermal titration calorimetry and microscale thermophoresis. TNBC cell lines MDA-MB-231 and MDA-MB-468 and a human TNBC xenograft model were used to verify in vitro and in vivo efficacy of the small molecule, respectively.

Results: We found that the small molecule, CRL1101, bound specifically to RelA as indicated by the biophysical assays. Further, CRL1101 blocked RelA nuclear translocation in breast cancer cells in vitro, and markedly reduced breast tumor growth in a triple-negative breast cancer xenograft model.

Conclusion: Our study demonstrates that CRL1101 may lead to new NF- $\kappa$ B-targeted therapeutics for TNBC. Further, blocking of nuclear translocation of shuttling transcription factors may be a useful general strategy in cancer drug development.

Keywords: transcription factors, breast cancer, computer aided drug design, nuclear transport, drug-target

\section{Introduction}

Triple-negative breast cancer (TNBC), a clinical breast cancer subtype lacking estrogen receptor (ER), progesterone receptor (PR) and overexpression of Her2, is highly proliferative but more sensitive to systemic chemotherapies. However, patient outcomes of TNBC are poor compared to the prognosis of other subtypes of breast cancer. While $93 \%$ of Her $2+$ breast cancer patients remain in remission for 5 years, this is only true for $77 \%$ of those individuals with TNBC. Although immunotherapies are showing promise in the treatment of TNBC, chemotherapy remains a standard approach. ${ }^{1}$ Nonetheless, for $20-30 \%$ of patients, there is a high risk for relapse within 3 years even after resection of primary tumors and chemotherapy. ${ }^{2}$
Correspondence: Ramachandran Murali Email ramachandran.murali@csmc.edu 
The NF- $\mathrm{KB}$ transcription factor is a central mediator of cell function and fate. Numerous studies have shown that $\mathrm{NF}-\kappa \mathrm{B}$ plays a critical role in promoting breast tumor growth, progression, and resistance to drug treatment. ${ }^{3}$ NF- $\mathrm{KB}$ can also be activated by multiple stimuli that may sometimes reduce the efficacy of treatments. For example, obesity is considered as a general risk-factor for breast cancer. In fact, for TNBC patients, studies show that adipose tissues elicit inflammatory cytokines such as $\mathrm{TNF} \alpha$ in a RelA dependent manner, ${ }^{4,5}$ and reduce the efficacy of cancer treatment. ${ }^{6,7}$ In another study, Wee et al. $^{8}$ showed that resistance to paclitaxel treatment in TNBC is conferred by an overexpression of interleukin-1 receptor associated kinase 1 (IRAK1) in response to NF$\kappa \mathrm{B}$-mediated cytokine production.

The activation of NF- $\mathrm{KB}$ can occur in response to different stimuli such as radiation, chemo-agents, and pro-inflammatory cytokines. ${ }^{9} \mathrm{NF}-\mathrm{\kappa B}$ can also be activated at different stages of tumor progression and can facilitate tumor growth. We and others recently observed that resistance to Trastuzumab leads Her2+ breast cancer cells to display the phenotypes of TNBC through the activation of NF- $\kappa B .{ }^{10,11}$ TNBC cells are known to harbor constitutive activation of NF- $\mathrm{kB},{ }^{12,13}$ which is implicated in poor prognosis for TNBC patients.

$\mathrm{NF}-\kappa \mathrm{B}$ is a protein complex consisting of Rel family proteins (RelA, RelB and cRel) located in the cytoplasm, where they reside in a resting state when binding to I $\kappa \mathrm{B}$ proteins $(\mathrm{I} \kappa \mathrm{B} \alpha, \mathrm{I} \kappa \mathrm{B} \beta)$, which masks the nuclear translocation signal in Rel proteins and thus prevents their DNA binding. Upon activation, I $\mathrm{I} B$ proteins are degraded through phosphorylation by IאB kinases (IKK). Rel proteins in complex with $\mathrm{p} 50 / \mathrm{p} 52$ proteins are then translocated to the nucleus where they bind to DNA. ${ }^{14} \mathrm{NF}-\mathrm{\kappa B}$ activation can occur through either canonical or non-canonical signaling. In canonical signaling, RelA is translocated into the nucleus, whereas in non-canonical signaling RelB/cRel are translocated.

Much effort has been directed to develop specific NF$\kappa B$ inhibitors. NF- $\kappa B$ activation can be blocked by interference at any stage of the NF- $\mathrm{KB}$ signaling cascades: upstream regulators, IKK complex, NF- $\mathrm{BB}$ nuclear translocation, DNA binding, transactivation, and post-translational modification. However, the only regulated step in the activation process is the phosphorylation of I $\mathrm{B}$ by IKK. ${ }^{15}$ Given IKK's central role in signal integration and relay in regulating $\mathrm{NF}-\kappa \mathrm{B}$ activation, I $\mathrm{B}$ kinases have been studied to develop NF- $\mathrm{B}$ signaling inhibitors.
However, decades of work has not yielded a clinically useful IKK inhibitor.

Recently, using TNBC tissues, Kim et al. ${ }^{16}$ have shown that among Rel proteins, a disproportionate accumulation of RelA in the cancer cell nucleus indicated poor prognosis. These observations, consistent with the established role of NF- $\mathrm{kB}$ in TNBC, further suggest that dysregulation of RelA shuttling between cytoplasm and nucleus is essential for TNBC progression. Thus, small molecules that are aimed at sequestering RelA in the cytoplasm and thereby blocking its DNA binding and transactivation may effectively silence anti-apoptotic gene expression.

Umezawa developed an irreversible RelA inhibitor, DHMEQ, that shows anti-cancer activity in leukemias. ${ }^{17}$ This is the only RelA inhibitor developed thus far. However, this irreversible inhibitor binds to a free cysteine (free-Cys) at position 38 located in the DNA binding domain of RelA. ${ }^{18}$ Since this free-Cys in the DNA binding domain is conserved among all three REL proteins in the NF- $\kappa$ B complex (Figure S1), we surmised that DHMEQ will be non-specific and likely block cRel, RelB and RelA. Thus, we hypothesized that preventing RelA nuclear translocation by an allosteric inhibitor will be an alternate approach to inhibiting constitutively active NF- $\mathrm{KB}$, rather than using RelA-DNA binding inhibitors or IKK inhibitors. Here, we show that a small molecule designed by a structure-based approach disabled the nuclear translocation of the RelA protein. The inhibitor blocked activation of NF- $\mathrm{kB}$ and its target genes, and inhibited xenograft tumor growth. Thus, our strategy to block RelA using an allosteric inhibitor may be relevant for cancer therapy and represents a novel approach to specifically target NF- $\mathrm{KB}$ activation.

\section{Materials and Methods Cell Lines and Reagents}

Spontaneously immortalized mouse embryo fibroblasts (MEFs) were obtained from Rodent transgenic core facility at Cedars-Sinai and maintained in DMEM (Invitrogen, Carlsbad, CA) supplemented with $10 \%$ donor calf serum (Invitrogen) and $100 \mathrm{U} / \mathrm{mL}$ penicillin and $100 \mathrm{mg} / \mathrm{mL}$ streptomycin.

Human triple-negative breast cancer cell lines MDAMB-231 and MDA-MB-468 were obtained from ATCC. These TNBC cells are highly proliferative, lack the expression of hormone receptors ER (estrogen receptor) and PR (progesterone receptor), and do not overexpress 
the Her2 receptor. Cells were grown in the Roswell Park Memorial Institute (RPMI)-1640 (Mediatech, Manassas, VA, USA) supplemented with $10 \%$ donor calf serum (Invitrogen), 100U/mL penicillin and $100 \mathrm{mg} / \mathrm{mL}$ streptomycin (Invitrogen). Both cells were incubated at $37^{\circ} \mathrm{C}$ in a humidified atmosphere containing 5\% $\mathrm{CO}_{2}$. CRL1101 was purchased from Chembridge, Inc. (San Diego, CA).

\section{Bioinformatics Analysis of TCGA Database}

The RNA-Seq data of Breast invasive carcinoma (BRCA) was obtained from the Broad Institute GDAC Firebrowse (http://firebrowse.org), which contains The Cancer Genome Atlas (TCGA) data version 2016_01_28. We downloaded the data of mRNAseq preprocess level 3 and used the raw counts of UNC RNASeqV2 in our study. The clinical information and PAM50 subtypes of the TCGABRCA dataset were gathered from the released dataset of Netanely et al. ${ }^{19}$ by Shamir lab. The clinical subtypes were then defined using estrogen receptor (ER), progesterone receptor (PR) and Her2 status. We matched sample IDs from the two resources and obtained 1031 tumor samples and 111 normal samples for our bioinformatics analysis. DESeq $2^{20}$ was applied to normalize the raw counts of RNA-seq data after filtering out low abundance genes, and differential expression analysis was performed. The $p$-values of multiple tests were adjusted using BenjaminiHochberg's method. ${ }^{21}$

\section{Design and Development of RelA Inhibitor}

The compounds have been designed using a stepwise procedure that identifies pseudo-allosteric cavities used to induce allosteric modification (CIAM) as described previously $^{22,23}$ to disable protein-protein interaction. The procedure has been modified to constrain ligand-induced structural changes in a protein complex; these steps involve identification of rigid cavity, virtual screening using GLIDE (Schrodinger, Inc.) and molecular simulations using the DESMOND function in Schrodinger (San Diego, CA, USA). Briefly, the three-dimensional structures of RelA dimer-DNA complexes (PBD code: $2 \mathrm{RAM}^{24}{ }^{24} \mathrm{NFI}^{25}$ and $1 \mathrm{VKX}^{26}$ ) were used as template. Computational analysis was performed at different temperature and simulation times to identify critical sites (S276 and NLS) to disrupt the nuclear localization signaling region (NLS) located between DNA-binding domain and transactivation domain (TAD) at the C-terminus. We have screened chemicals from Chembridge database $(>$ 500,000 compounds) that are filtered for drug-like properties that can disrupt NLS motif structural disposition thereby preventing RelA binding to DNA. CRL1101 was identified as a potential inhibitor for biological characterization. Structure analysis and modeling were performed using software from SBGRID. ${ }^{27}$ The calculations were performed at Cedars-Sinai high-performance computing center facility.

\section{Expression and Purification of Recombinant RelA}

Human RELA encoding amino acid residues 20-321 was cloned into pET-21(a) vector and expressed as a C-terminal His-tag fusion protein in BL21 (DE3) cells. The cells were resuspended in $1 \mathrm{x}$ phosphate buffered saline (PBS), sonicated and soluble lysate was passed through a Ni-NTA $5 \mathrm{~mL}$ FF column (GE Healthcare) equilibrated in buffer A (20 mM Tris pH 7.4, $500 \mathrm{mM}$ $\mathrm{NaCl}$ ). The column was washed and eluted with buffer $\mathrm{A}$ supplemented with $40 \mathrm{mM}$ and $400 \mathrm{mM}$ Imidazole, respectively. The sample was dialyzed against $20 \mathrm{mM}$ Tris $\mathrm{pH} 7.4$ and put through a SP sepharose $5 \mathrm{~mL}$ FF column (GE Healthcare). The protein was eluted using 0 to $300 \mathrm{mM} \mathrm{NaCl}$ gradient, dialyzed against PBS $+15 \mathrm{mM}$ $\beta$-ME, concentrated and stored at $-80^{\circ} \mathrm{C}$.

\section{Isothermal Titration Calorimetry (ITC)}

The binding thermodynamics of RelA to the CRL1101 was measured by ITC using a high precision VP-ITC titration calorimetric system (Microcal LLC, Northampton, MA). The calorimetric cell containing RelA at a concentration of $6-10 \mu \mathrm{M}$ dissolved in $10 \mathrm{mM}$ Tris (pH 8.0), 1mM EDTA was titrated with CRL1101 at a concentration ranging from 400-600 $\mu \mathrm{M}$. Injection volumes were $10 \mu \mathrm{L}$. The heat evolved upon each injection of CRL1101 were obtained from the integral of calorimetric signal. The heat associated with binding of CRL1101 to RelA was obtained by subtracting the heat of dilution from the heat of reaction. The measurements were made at $25^{\circ} \mathrm{C}$. Data were analyzed and fitted by using the data analysis software supplied by Microcal (ORIGIN 5.0). The free energy generated by RelA binding to CRL1101 was estimated from $\Delta \mathrm{G}=-\mathrm{RT} \ln \mathrm{K}=$ $\Delta \mathrm{H}-\mathrm{T} \Delta \mathrm{S}$. 


\section{Ligand Binding by Microscale} Thermophoresis

Monolith NT.115 Microscale Thermophoresis (MST) instrument (Nanotemper Technologies) was used for this assay. Monolith protein labeling kit RED-NHS was purchased from Nanotemper Technologies. Briefly, recombinant RelA protein was labeled using RED-NHS 2nd generation labelling kit (NanoTemper) following manufacturer's instructions. A serial dilution of ligand CRL1101 $(0.25 \mathrm{mM}$ to $0.00763 \mu \mathrm{M})$ was prepared and titrated against $0.55 \mu \mathrm{M}$ labeled RelA. The assay was read in $1 \%$ excitation power and $40 \%$ of MST power.

\section{Nuclear Localization of RelA in TNBC Cell Lines by Fluorescent Microscopy}

Cells were treated with or without $15 \mu \mathrm{M}$ of CRL1101 in $1 \%$ dimethyl sulfoxide (DMSO). 45 min after treatment, cells were fixed with $4 \%$ formaldehyde for $10 \mathrm{~min}$ and sequentially treated with $0.1 \%$ Triton X-100 for $10 \mathrm{~min}$. Cells were blocked in $1 \%$ bovine serum albumin (BSA) in phosphate buffered saline (PBS) for $10 \mathrm{~min}$. Cells were incubated with anti-RelA(p65) antibody (1:150, Abcam, Cambridge, UK) in PBS with $1 \%$ BSA overnight at $4{ }^{\circ} \mathrm{C}$ and a secondary antibody (1:1000 anti-rabbit Alexa 488, Invitrogen, Carlsbad, CA, USA) for $1 \mathrm{~h}$ in the dark. Analyses were performed using a microscope (Nikon ECLIPSE Ti-U, Nikon, Tokyo, JAPAN). Green signal shows RelA(p65), blue signal shows 4',6-diamidino-2phenylindole (DAPI).

\section{Cell Viability Assay}

Cell viability was quantitated using a colorimetric MTT assay according to the procedures described previously. Briefly, $100 \mu \mathrm{L}$ of target cell suspension ( $1 \times 10^{4}$ cells) were added to each well of 96 -well plate, and the plate was incubated for $24 \mathrm{~h}$ at $37{ }^{\circ} \mathrm{C}$ in a humidified $5 \% \mathrm{CO}_{2}$ atmosphere. Following incubation, $10 \mu \mathrm{M}$ of MTT working solution was added to each well, and the plates were incubated for $3 \mathrm{~h}$ at $37^{\circ} \mathrm{C}$. After addition of inhibitor, the absorbance values were measured with a microplate reader at $570 \mathrm{~nm}$. The percentage of survival was calculated using the following formula: survival percentage $=$ (absorbance of CRL1101 treated wells - blank wells/absorbance of untreated wells - blank wells) X 100. The inhibition constant $\left(\mathrm{IC}_{50}\right)$ of the inhibitor was obtained by regression analysis using Excel.

\section{Western Blot Analysis}

Expression and phosphorylation changes of NF- $\mathrm{KB}$ related molecules after CRL1101 treatment were examined by Western blot. Western blot analysis was performed to

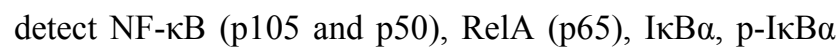
and $\beta$-actin protein expression. $\beta$-actin was used as a loading control. Time course for $0-60 \mathrm{~min}$ after $15 \mu \mathrm{M}$ of CRL1101 treatment was examined.

\section{Anchorage Independent Growth Assay}

The effects of CRL1101 on clonogenic survival were analyzed. Base layers consisting of growth medium containing $0.53 \%$ low-melting point agarose (Invitrogen) were poured onto 6-well plates and allowed to solidify. Cells were seeded at concentration of 10,000 cells/well in triplicate in top layers consisting of growth medium containing $0.32 \%$ agarose. After $24 \mathrm{~h}, 15 \mu \mathrm{M}$ of CRL1101 was added. Cells were incubated for 14 days to form visible colonies. The colonies were fixed and stained by $0.05 \%$ Crystal violet in 50\% methanol.

\section{NF- $\kappa B$ Reporter Assay}

MDA-MB-231 cells transfected with the pGL3-luciferase

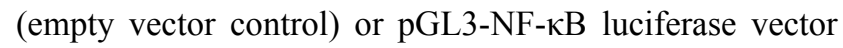
were treated with $10 \mu \mathrm{M}$ CRL1101 or DMSO vehicle for $24 \mathrm{~h}$ the day after transfection. $10 \mu \mathrm{M}$ CRL1101 was used in this assay instead of $15 \mu \mathrm{M}$ due to reduced cell viability. Cells were grown in $10 \%$ FBS medium. Reporter activity was measured by luciferase assays and was normalized to $\beta$-galactosidase activity.

\section{Cell Migration Assay}

Cells were seeded in 6-well plates at $1 \times 10^{5}$ per well and treated with either vehicle or $10 \mu \mathrm{M}$ CRL1101 (of note, 10 $\mu \mathrm{M}$ was used due to reason stated above). A line was drawn on the underside of the well with a pipette tip. Cell migration was assessed by measuring the distance between wound edges. Migration results are expressed as the average migration distance $(\mu \mathrm{m} \pm \mathrm{SD})$. The cells were monitored by phase contrast microscopy on an inverted microscope. All the data presented are from at least 3 independent experiments performed in duplicate.

\section{Tumor Growth Study}

NCr homozygous athymic (nude) mice (eight weeks-old) were purchased from Charles River Laboratories. An aliquot of $2 \times 10^{6}$ MDA-MB-231 cells were suspended in 200 
$\mathrm{mL}$ of PBS and injected subdermally in the right thigh of each animals. When xenograft tumors reached $\sim 200$ $230 \mathrm{~mm}^{3}$ in volume, animals were regrouped into two treatment groups $(n=6)$ : Vehicle control and CRL1101 alone. After tumor cell engraftment when tumor size is palpable, on day 9, mice were treated with CRL1101 $(25 \mathrm{mg} / \mathrm{kg})$ intraperitoneally every other day. Animals were maintained in accordance with guidelines of the Institutional Animal Care and Use Committee (IACUC) of Cedars-Sinai Medical Center. Tumor growth was monitored three times weekly for four weeks. Tumor volume was calculated by the formula: $\pi / 6 \mathrm{x}$ (larger diameter) $\mathrm{x}$ (smaller diameter) ${ }^{2}$. Mice body weight during the treatment was measured every 2 days.

\section{Statistical Analysis}

All experiments were repeated independently a minimum of three times and expressed as mean values with $95 \%$ confidence. The activity of CRL1101 was analyzed using cell viability assay and assessed by paired $t$-test. The other results were assessed by one-way ANOVA following by Scheffe's F-test. A value of $p<0.05$ was considered to indicate statistical significance.

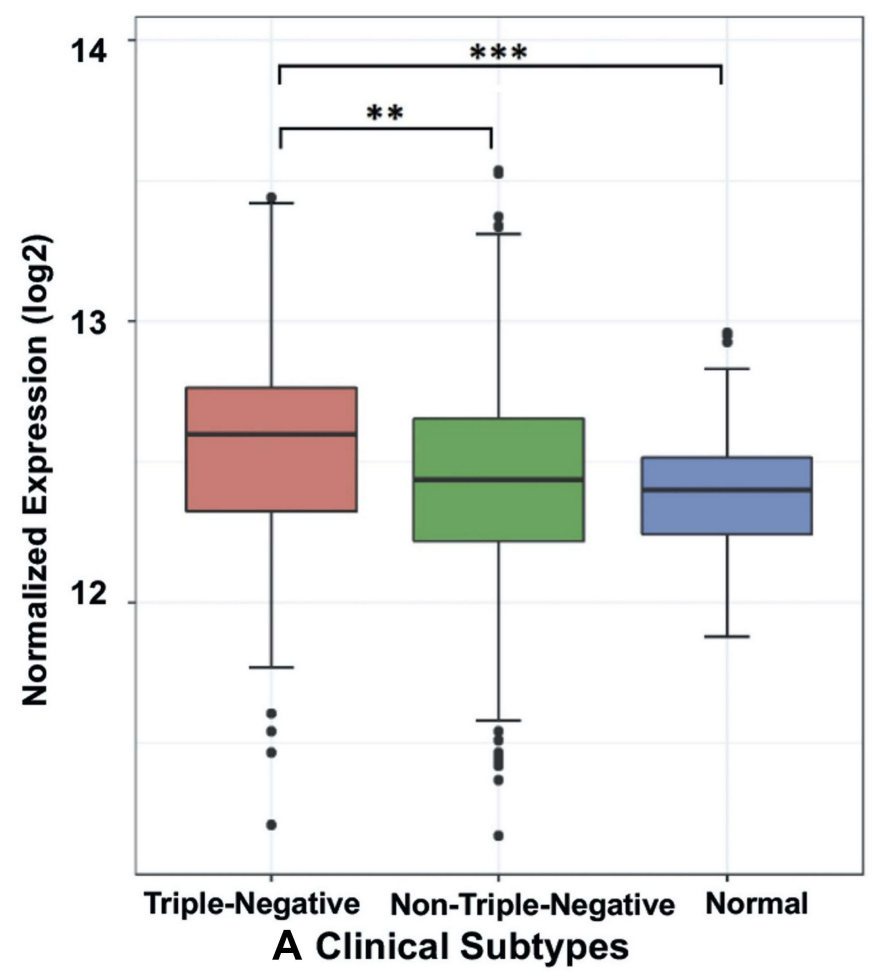

\section{Results}

\section{RelA is Overexpressed in TNBC Compared to Other Breast Cancer Subtypes}

$\mathrm{NF}-\kappa \mathrm{B}$ is constitutively activated in TNBC and plays an important role in cancer cell survival and resistance. ${ }^{12,28}$ The activation of NF- $\mathrm{KB}$ is also critical for the function of breast cancer stem cells $(\mathrm{BCSC})^{29}$ that promotes tumor re-emergence and metastasis. In either neoadjuvant or adjuvant settings, certain chemotherapeutic agents activate NF- $\mathrm{kB}$. Indeed, our TCGA data analysis show that the expression of RelA is significantly higher in TNBC than in normal breast tissue and other breast cancer subtypes (Figure $1 \mathrm{~A}$ and $\mathrm{B}$ ). The results are consistent with observation made by others using breast cancer tissues. Jones et al. observed nuclear NF- $\kappa B$ in TNBC tissues by immunohistochemistry (IHC) in response to chemotherapy correlated with high-grade tumor. ${ }^{30}$ Similarly, Wang et al. ${ }^{31}$ showed overexpression of RelA is associated with TNBC by interfering with celecoxib mediated apoptosis. These studies show overexpression of RelA, as measured by mRNA levels and

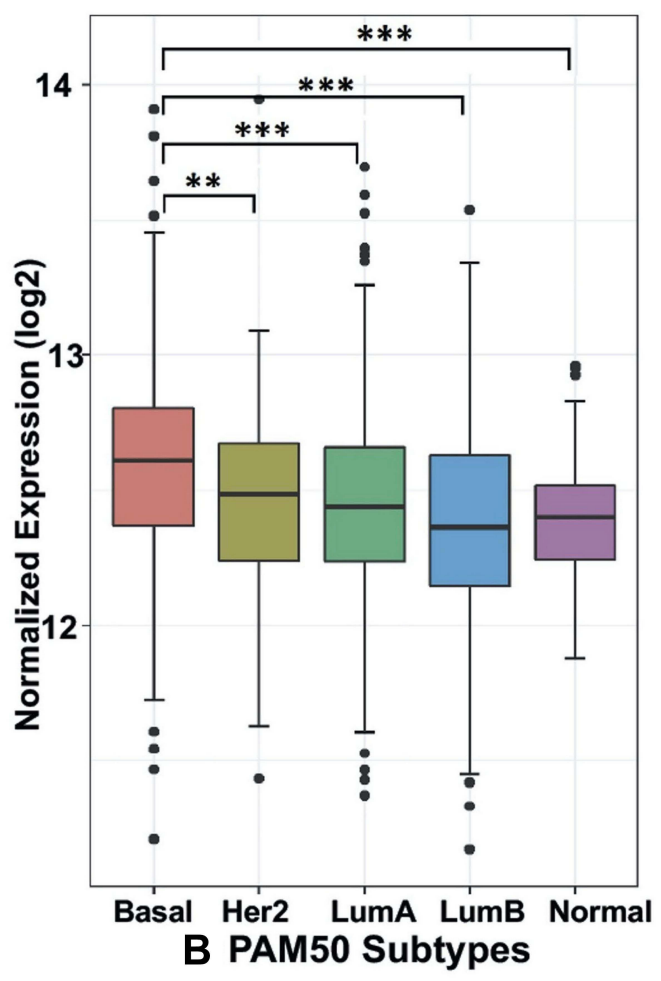

Figure I The boxplot of gene expression of RELA in breast cancer from TCGA: (A) clinical tumor subtype and (B) PAM50 subtype. The gene expression is presented as normalized counts using DESeq2 with variance stabilizing transformations (VST) on log2 scale. The comparison results between subtypes are presented as $* * \mathrm{P}<0.0 \mathrm{I}$, *** $\mathrm{P}<$ 0.00 I; 2-tailed unpaired $t$ test with Bonferroni correction. 
constitutive expression of nuclear RelA, promotes cancer growth and progression in TNBC. Furthermore, using TNBC tissues, Kim et al. ${ }^{16}$ have shown that among Rel proteins, an accumulation of nuclear RelA in the cancer cells indicated poor prognosis. Hence, we hypothesize that blocking RelA transported from cytoplasm into nucleus will limit tumor growth.

\section{Design and Development of RelA Inhibitors by a Structure-Based Approach}

We used a structure-based approach to test whether a small molecule can be developed to block the nuclear translocation of RelA. In the inactive state of $N F-\kappa B$, the RelA-p50-IкB $\alpha$ protein complex is located in the cytoplasm. In the active state, phosphorylation of $\mathrm{I} \kappa \mathrm{B} \alpha$ leads to its degradation, resulting in RelA-p50 translocation to the nucleus. The crystal structure of RelA complexed with p50 and $\mathrm{I} \kappa \mathrm{B} \alpha$ (Figure $2 \mathrm{~A}$ ) was determined by Jones et al. $^{25}$ and showed that the inactive complex is held together through a network of hydrogen bonds that keep the NLS masked by p50 (Figure S2). During this process, the nuclear localization signal (NLS), located after the DNA binding domain in C-terminus of RelA, drives nuclear translocation of

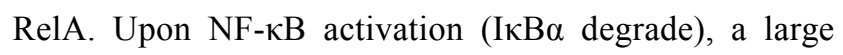

conformational change (relaxed by the network of hydrogen bonds) exposes the nuclear localization signal (NLS) motif (Figure 2B).

Previously, we used CIAM to identify allosteric sites in protein. ${ }^{22,23}$ Using the modified approach we identified a distal inhibitor-binding site from the NLS motif (Figure 2A and B) proximal to S276, a known phosphorylation site. To block nuclear translocation of RelA, structural changes in RelA must be restrained so that NLS motif remains unchanged. For this purpose, the inhibitorinduced conformational changes (ie, network of hydrogen bonds shown in Figure S2) need to be restrained. Hence, rigidity of the cavity was assessed by monitoring cavity size calculated using sitemap (Schrodinger, Inc) from molecular simulation results. Binding site proximal to S276 (Figure 2C) (cavity volume $442 \AA^{3}$ and Dscore $=0.955$ ) was used from an average structure from the molecular simulation for subsequent virtual screening. We selected 15 compounds based on change in binding energy ( $\Delta \mathrm{G}$ bind ranging from $3-8 \mathrm{Kcal} / \mathrm{mol}$ ) from MMGBSA analysis. One of the compounds, CRL1101 (Figure $2 \mathrm{D})$ was identified $(\Delta \mathrm{G}$ bind $=7.8 \mathrm{Kcal} / \mathrm{mol}$ ) as the most potent compound based on the combination of breast cancer cell proliferation assays and differential scanning fluorimetry (DSF). ${ }^{32}$
A

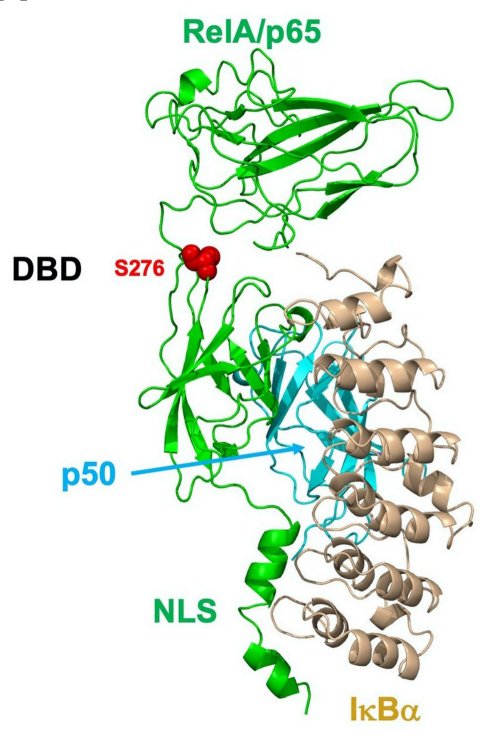

B

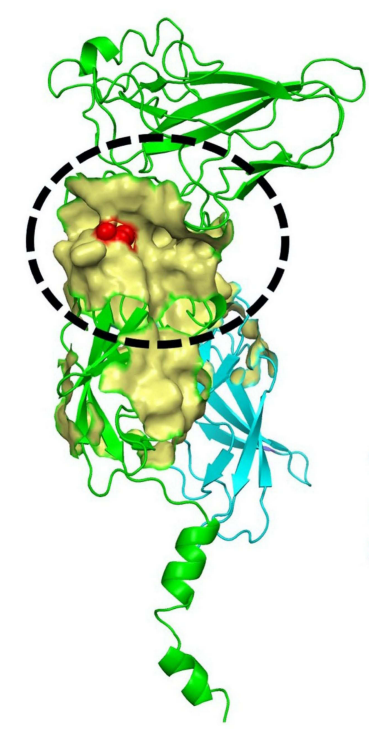

C

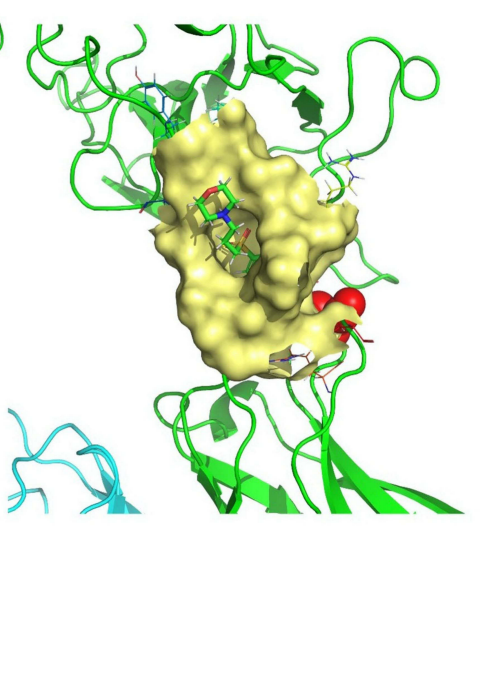

D

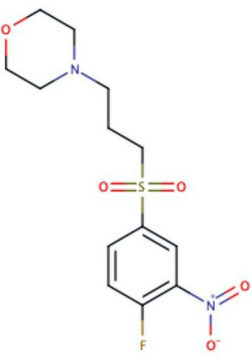

Figure 2 Three-dimensional structure of RelA with putative binding of CRLII0I: (A) Resting state of RelA (green) is found in complex with pI05 ( $\mathrm{p} 50$ is shown (cyan)) and the Ankyrin repeat of $1 \kappa B \alpha$ (light brown color) which sequesters the NLS of RelA preventing nuclear translocation of RelA-p50 protein complex. The DNA binding domain (DBD) is located at the N-terminus, while the nuclear localization signal (NLS) motif is located at the C-terminus. Key phosphorylation site, S276 is shown as red sphere model (B) Inhibitor-binding pocket is show in surface representation (Yellow). Location of S276 is shown in red (C) Closeup look at the binding of CRLI I0I (shown in stick representation; carbon, nitrogen and oxygen are shown in green, blue and red colors, respectively) binding to RelA (yellow surface). Partial structure of p50 is shown in cyan color (D) Chemical structure of CRLIIOI is shown. Pictures were created using Pymol. ${ }^{27,46}$ 


\section{CRLIIOI Inhibits Nuclear Translocalization of RelA}

Next, we tested if CRL1101 is specific for RelA. For this purpose, we used RelA-null cells. Cells treated with CRL1101 were unaffected based on cell survival assays compared to the wild-type suggesting that CRL1101 is specific to RelA. (Figure 3A). Furthermore, to check whether CRL1101 can sequester RelA in the cytoplasm, two TNBC cell lines, MDA-MB-231 and MDA-MB-468 were treated with $15 \mu \mathrm{M}$ of CRL1101. Immunofluorescence showed that CRL1101 diminished nuclear localization of RelA (Figure 3B).

\section{Biophysical Characterization of CRLI I0 I Directly Binding to RelA}

We wanted to verify whether CRL1101 could directly bind to RelA protein. First, we tried to measure the binding of CRL1101 by Surface Plasmon Resonance (SPR). However, the resonance signal was insufficient to measure kinetic parameters. We reasoned that immobilization of RelA might be interfering with ligand binding. Hence, we tried two techniques that do not require immobilization: (1) In microscale thermophoresis (MST) assay there is no immobilization involved and both ligand and target can freely move in the favored buffer. In this technique either target or ligand can be labeled. Here, we labeled the target protein RelA with fluorescent dye. CRL1101 bound to RelA with a binding constant $\left(K_{\mathrm{D}}\right)$ of $2.3 \mu \mathrm{M}$ (Figure 4A). (2) Similar to MST, in isothermal titration calorimetry (ITC), protein and ligand are free and provide information related to thermodynamics of the interaction.
In this assay, we observed CRL1101 binding to recombinant RelA with an affinity of $2.2 \mu \mathrm{M}(\Delta \mathrm{H}=-1734 \mathrm{Kcal} / \mathrm{mol}$ and $\Delta \mathrm{S}=14.1 \mathrm{cal} / \mathrm{mol} / \mathrm{deg} ; \mathrm{N}=2$ ) (Figure 4B) confirming direct binding of CRL1101 to RelA.

\section{Biological Activity of CRLIIOI}

Having demonstrated that CRL1101 specifically binds to RelA, we tested its biological activity in a series of in vitro assays. First, we measured the effect of CRL1101 on cell proliferation in TNBC breast cancer cell-lines, MDA-MB231 and MDA-MB-468. IC ${ }_{50}$ of CRL1101 in MDA-MB231 and MDA-MB-468 was $12.76 \mathrm{mM}$ and $12.33 \mathrm{mM}$ respectively. As expected, CRL1101 significantly inhibited cell proliferation with an inhibition constant of $15 \mu \mathrm{M}$ in both cell lines (Figure 5A). Next, we tested whether CRL1101 can block constitutive activation of NF- $\kappa$ B. Indeed, CRL1101 inhibited NF- $\kappa \mathrm{B}$ activation as measured by decrease in phosphorylation of $\mathrm{I} \kappa \mathrm{B} \alpha$ in both tumor cell lines (Figure 5B). However, there was a small reduction in expression of RelA in MDA-MB-231 at the 60 min time point as opposed to MDA-MB-468. The significance of this reduction of RelA expression in MDA-MB-231 is yet to be determined. Next, we examined the CRL1101 effect on colony formation. As shown in Figure 5C, CRL1101 inhibited colony formation. These results show that CRL1101 limits tumor cell growth.

Using a NF- $\kappa \mathrm{B}$-luciferase vector which contains tandem RelA-binding sites in the promoter governing the luciferase gene, we also found that CRL1101 inhibited the activity of luciferase reporter induced by RelA binding in MDA-MB-231 cells (Figure 6A). Next, we checked whether CRL1101 affected RelA-regulated gene
A

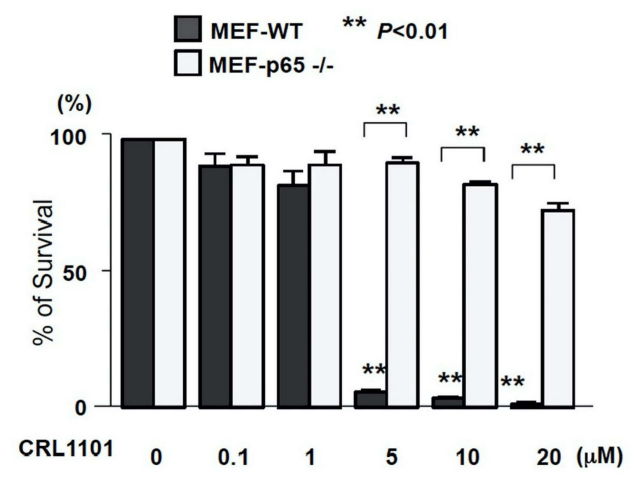

B

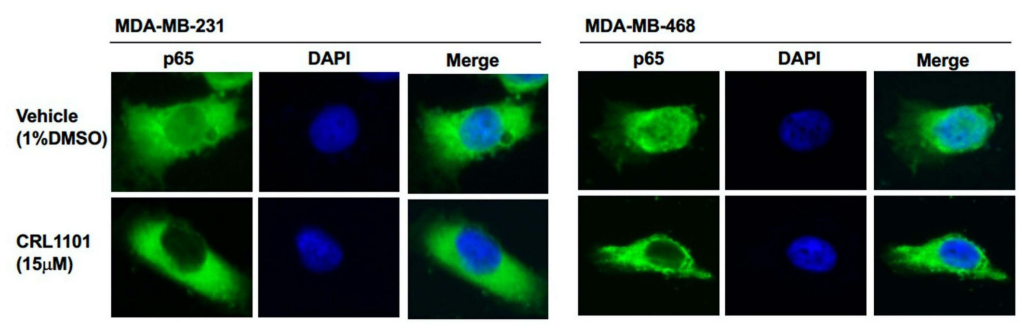

Figure 3 CRLIIOI is specific to RelA: (A) CRLIIOI affected RelA-null cells viability. Cell survival of RelA-null MEF cells were measured by MTT assay after treatment of CRLIIOI at different concentrations. ${ }^{* * P}<0.01$ (B) CRLIIOI sequestered RelA in the cytoplasm. Localization of RelA in TNBC breast cancer cells, MDA-MB-23I and MDA-MB-468, measured by fluorescence microscopy. TNBC cells treated with CRLI I0I retained RelA (p65, green) in the cytoplasm compared to cells treated with vehicle. Cellular nuclei are stained with DAPI (blue). 

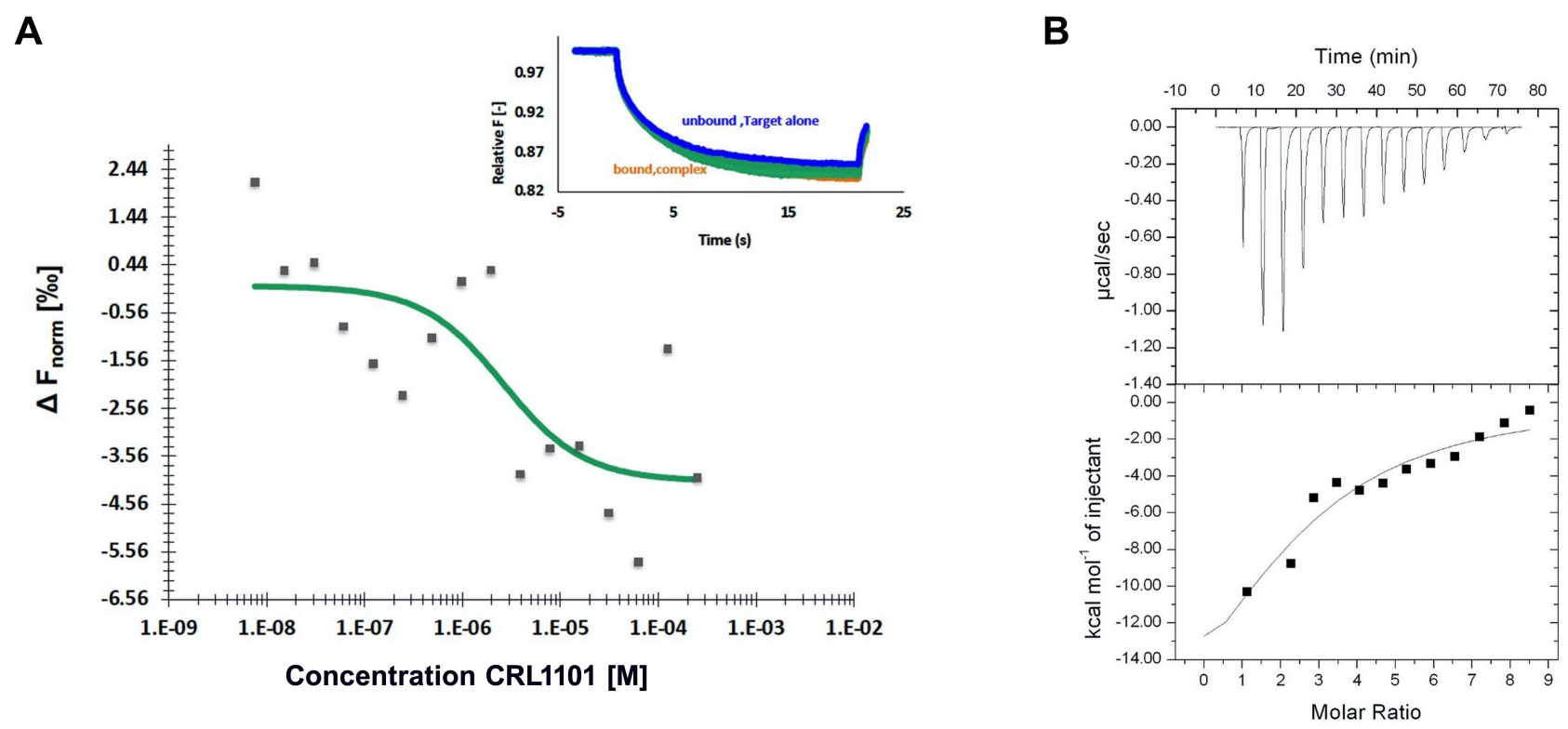

Figure 4 Analysis of CRLI I0I binding to recombinant RelA: (A) Microscale Thermophoresis technique (MST) Dose response curve upon titrating CRLI0I I from 0.0076 to $250 \mu \mathrm{M}$ against $0.55 \mu \mathrm{M}$ RelA. The $K_{\mathrm{D}}$ obtained was $2.3 \mu \mathrm{M}$. Insert represents MST traces depicting fluorescence change over time at different concentrations of CRLI0II. Traces corresponding to unbound and bound protein are shown in blue and green respectively. (B) Isothermal titration calorimetry: Serial dilution of IO $\mu M$ CRLI I0I was titrated against recombinant RelA and heat generated from the interaction is shown. Affinity of CRLIIOI to RelA is $2.2 \mu \mathrm{M}$.

A

MDA-MB-231

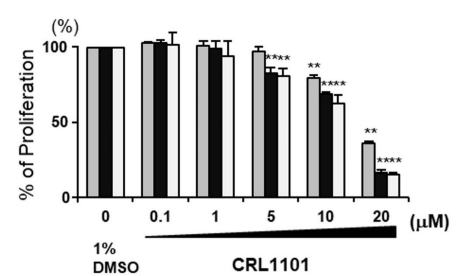

DMSO
MDA-MB-468

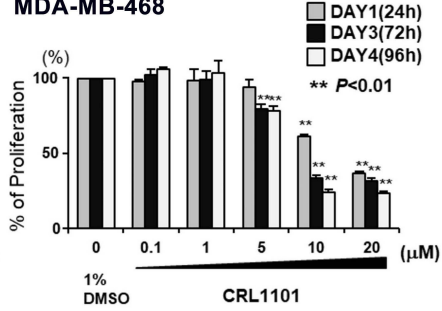

B
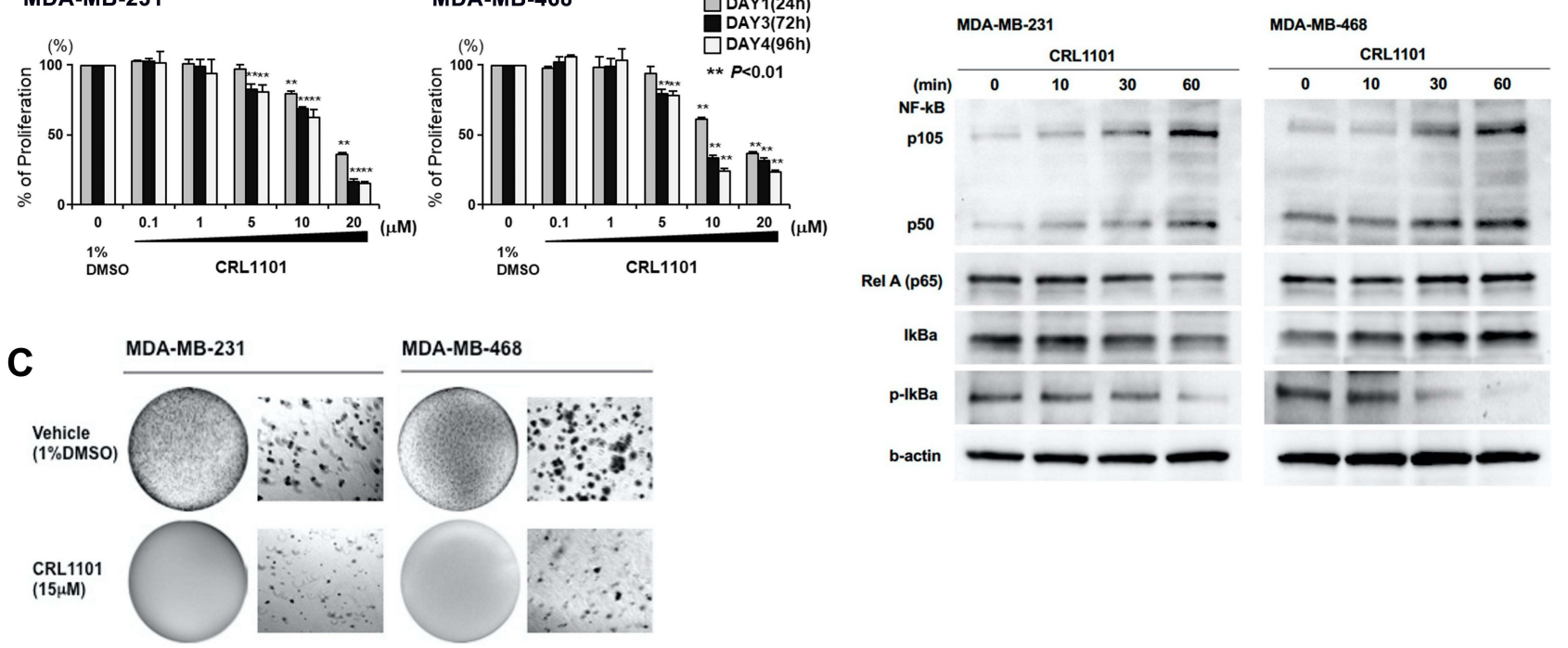

Figure 5 Anti-tumor effects of CRLIIOI in TNBC: (A) Anti-proliferation effects of CRLIIOI. Cells were treated with different concentations of CRLII0I, as shown in figure. Proliferation of the cells were investigated by MTT assay at 24,72 and $96 \mathrm{~h}$ after CRLII0I treatment. Proliferation was inhibited in a dose-dependent manner $(* * P<0.01)$. (B) Time series of the effects of CRLIIOI on expression and activation of NF- $\kappa B$ and related molecules by Western blot analysis. The effects of $15 \mu M$ of

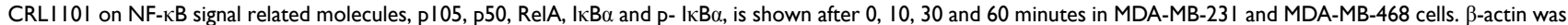
used as an internal expression standard. (C) Inhibition of tumor growth by anchorage independent growth assay. The effects of CRLIIOI on clonogenic survival is shown after 24 h. CRLIIOI inhibited colony formation in MDA-MB-23I and MDA-MB-468 cells after cells were treated with I5 $\mu$ M of CRLIIOI.

expression. In breast cancer, the expression of interleukin$8(\mathrm{IL}-8)^{33,34}$ and EZH2 has been shown to depend on RelA. $^{35}$ Thus, we examined the expression of IL8 and EZH2 in MDA-MB-231 cells treated with CRL1101. RTPCR showed that IL-8 and EZH2 mRNA levels were significantly reduced while p65 and p50 levels remained unaffected (Figure 6B). We also tested if CRL1101 at 10 $\mu \mathrm{M}$ can affect cell migration which is known to be regulated by NF-kB. In a wound-healing assay, CRL1101 showed reduced cell migration in TNBC cells (Figure 6C 
A

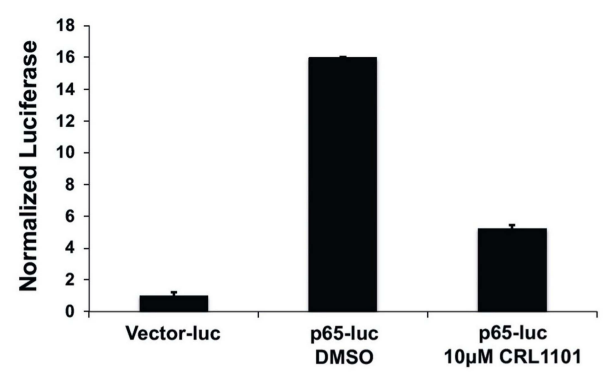

C

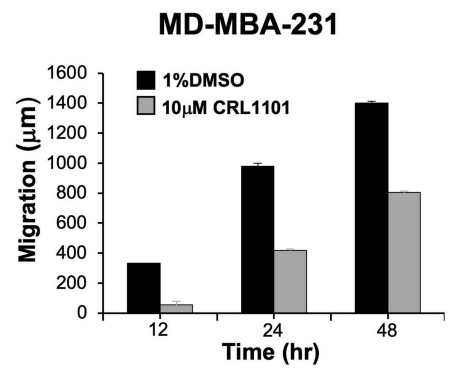

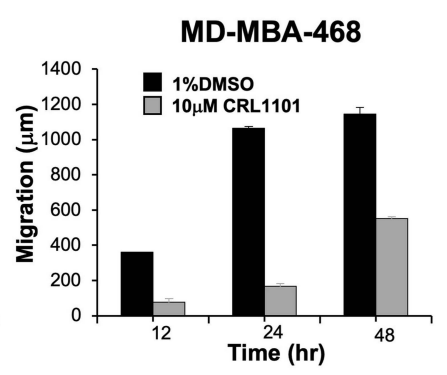

B

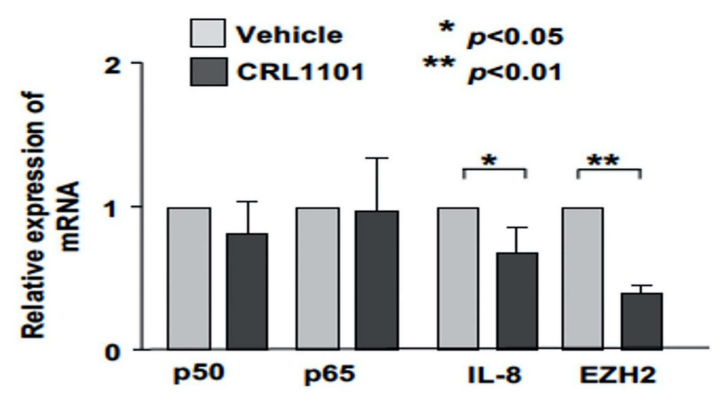

D

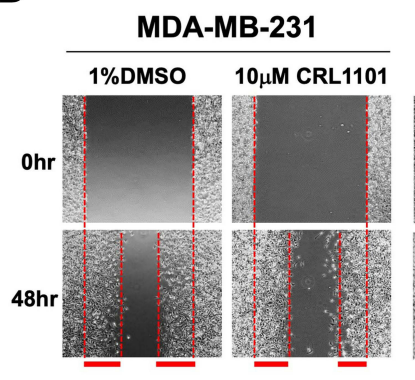

Figure 6 (A) Regulation of NF-KB-responsive reporter activity by CRLI I0 I: MDA-MB-23I cells transfected with the pGL3-luciferase (empty vector control) or pGL3-NF$\kappa B$ binding luciferase vector were treated with $10 \mu \mathrm{M} C R L I I 0 I$ or DMSO vehicle for $24 \mathrm{~h}$ the day after transfection. Reporter activity was measured by luciferase assays and was normalized to $\beta$-galactosidase activity. (B) RelA inhibitor, CRLI I0I reduced pro-inflammatory cytokines in MDA-MB-23 I cells. The inhibitory effects of CRLI I0I (5 $\mu$ M) on RelA-dependent gene regulation in MDA-MB-23I cells was assayed by using quantitative real time PCR. Two key players, IL8 and EZH2, regulated by nuclear RelA decreased after $48 \mathrm{~h}$ incubation with CRLI I0I. ${ }^{*} \mathrm{P}<0.05$ and $* * \mathrm{P}<0.0$ I (C) CRLI I0I diminish tumor cells' migration. Cells treated with CRLI I0I have reduced cell migration as compared to the vehicle control ( $1 \%$ DMSO). Migration results are expressed as the average migration distance $(\mu \mathrm{m} \pm \mathrm{SD})$. The cells were monitored by phase contrast microscopy on an inverted microscope at 12, 24 and $48 \mathrm{~h}$. All the data presented are from at least 3 independent experiments performed in duplicate. (D) Representative images for the wound healing assay. Control (I\% DMSO) and CRLI IOI (I0 $\mu \mathrm{M})$ treated MDA-MB-23I and MDA-MB-468 cells are shown at 0 and $48 \mathrm{~h}$. Verticle red lines are drawn along wound edges and migration distance is depicted as thick horizontal red lines.

and D). These results suggest that targeting RelA nuclear translocation impairs NF-kB-mediated cell function.

\section{Therapeutic Efficacy of CRLI IOI in a Pre- Clinical TNBC Mouse Model}

We examined the therapeutic efficacy of CRL1101 in a common TNBC xenograft model. ${ }^{36}$ Briefly, MDA-MB231 cells were used to grow tumors in athymic mice. Mice were treated with $25 \mathrm{mg} / \mathrm{kg} /$ day (IP) CRL1101 for 4 weeks. The control group was treated with vehicle. Mice treated with CRL1101 showed significant reduction in tumor growth (Figure 7A and B). At day 31, the CRL1101-treated group had reduced tumor volume by $50 \%$ compared to the PBS group $(P<0.001)$. During the treatment there was no significant change in body weight and the change was within the margin of error due to differences in tumor size. These results suggest that CRL1101 has a potent antitumor effect in vivo and does not show severe toxicity in mice (Figure 7C).

\section{Discussion}

RelA/p65 of NF-kB has been shown to promote cancer cell growth and resistance to therapy in many cancers including breast cancer. ${ }^{10,12,29}$ There have been many inhibitors developed against NF- $\mathrm{KB}$ that mainly target IKK. ${ }^{37}$ To date, clinical trials with NF- $\mathrm{KB}$ inhibitors have not been successful largely due to the essential role of the NF- $\mathrm{KB}$ family transcription factors in pleiotropic physiological functions and thus ubiquitous supression of total $\mathrm{NF}-\mathrm{\kappa B}$ activity leads to undesirable toxicity. ${ }^{38}$ In contrast, we demonstrated a novel way to disable constitutively activated NF- $\mathrm{KB}$ mediated tumor growth by targeting RelA, the dominant TNBC-associated NF- $\kappa B$ transcription factor subunit, using a small-molecule inhibitor to prevent its nuclear translocation.

RelA-specific inhibitors have not been developed. As mentioned before, the irreversible inhibitor, DHMEQ binds to Rel-family proteins and is not specific to RelA. $^{39}$ To develop a RelA-specific inhibitor we used a non-canonical approach. Previously, we developed a novel 
A

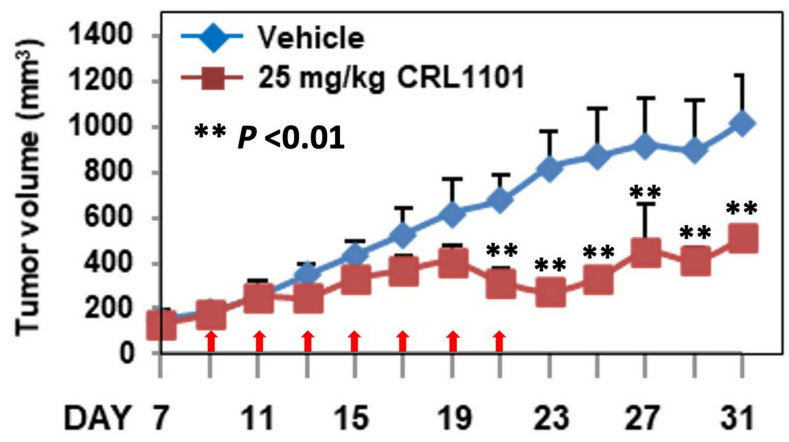

C

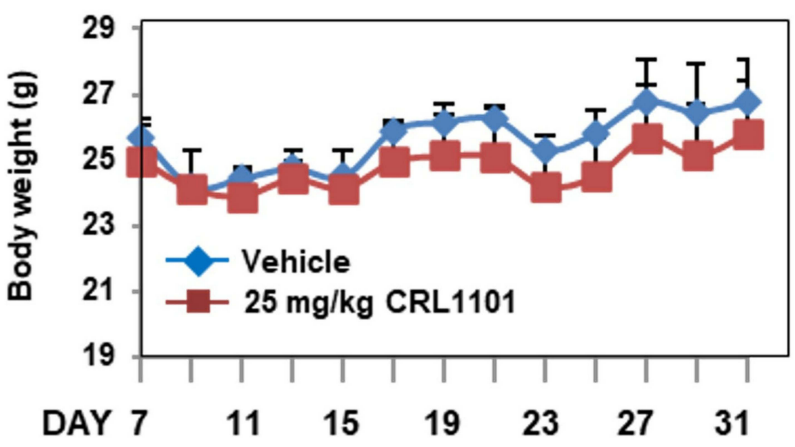

B

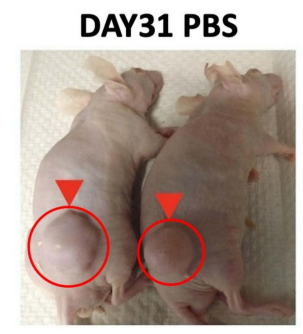

DAY31 CRL1101

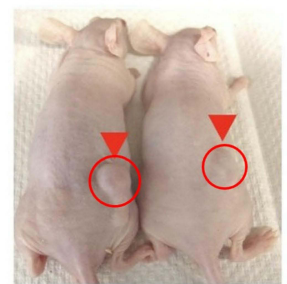

Figure 7 CRLIIOI limits tumor growth breast in a TNBC mouse model: Tumor growth mediated by MDA-MB-23I cells was assessed in athymic mice. (A) Mice were administered with $25 \mathrm{mg} / \mathrm{kg} /$ day of CRLI IOI or vehicle (PBS) by IP injection, as indicated by arrows. Results are given as mean tumor volume (mm ${ }^{3}$ ) \pm S.E. Treatment days are shown by arrows. Tumor growth in animals treated with CRLI I OI was significant ( $* * P<0.0 I)$ compared to vehicle. $N=6$. $(B)$ Visual of tumor size at the end of experiment is shown. (C) Body weight during the treatment is shown. Body weight during the treatment was measured and no significant loss of weight was observed.

computer algorithm termed "Cavity-induced allosteric modification (CIAM)", 40 This approach was used to disable protein-protein interactions such as TNFR-TNF $\alpha$ receptor complex, ${ }^{22}$ and survivin. ${ }^{23}$ The algorithm is based on the identification of molecular/atomic determinants responsible for flexibility in the protein molecule. The crystal structure of RelA/p50 protein complex revealed that large conformational and structural changes are needed for RelA nuclear localization. We exploited this structural aspect of RelA and identified CRL1101. In a series of in vitro and in vivo studies, we demonstrated that CRL1101 is a novel agent that binds to RelA with an affinity of $2.2 \mu \mathrm{M}$. CRL1101 is also specific to RelA based on the observation from RelA-null MEF cells. Of note, it has been shown that tumor cells lacking of either cRel or RelB show increased proliferation in tumor cells.41,42 Since we observed wild-type cells treated by the inhibitor undergo apoptosis, we believe CRL1101 is specific to RelA. We plan to explore the specificity of CRL1101 in next set of studies by expressing cRel and RelB recombinant proteins.

CRL1101 as an allosteric inhibitor shows anti-tumor effect in vitro and in vivo. Treatment of TNBC cells, MDA-MB-231 and MDA-MB-468, with CRL1101 also diminished nuclear localization of RelA (Figure 3B). By sequestering RelA in the cytoplasm, CRL1101 inhibited $\mathrm{NF}-\kappa \mathrm{B}$ activation and concomitantly reduced the expression of pro-metastatic genes such as IL-8 and EZH2, consistent with ability of CRL1101 to limit cell migration, suggesting that our molecule might also aid in delaying/ preventing cancer metastasis. This allosteric inhibitor reduced tumor growth in a breast cancer mouse model (Figure 7A) suggesting the biological effect observed is due to blocking RelA nuclear transport.

Activation of $N F-\kappa B$ is critical for adaptive/innate immunity. In this context, p50, RelB and cRel play a dominant role. Lack of expression of RelA in immune cells such as lymphocytes and macrophages has been shown to promote suppressive phenotype. It can be argued that CRL1101 can promote immunosuppresive environment in cancer. However, when we used CRL1101 in pancreatitis mouse model as an anti-inflammatory study using wild-type B6 mice, it was found that CRL1101 did not have any effect on immune cell function (ie, promote immunosuppression) based on the cytokine profiles (unpublished data, personal communication with Drs. Pandol and Edderkaoui). Thus, we believe that CRL1101 will have minimal or no effect on tumor microenvironment 
of cancer. However, effect of CRL1101 on T-cells or macrophage cannot be ruled out. This aspect requires further investigations that may involve targeted delivery of CRL1101 by nanoparticles or as drug-conjugates used by us before. ${ }^{43-45}$

In summary, we have developed a novel way to disable $\mathrm{NF}-\kappa \mathrm{B}$ activation by selectively targeting nuclear localization of RelA in breast cancer. This new approach will be a new tool to target cancer that depends on NF-кB function for growth and metastasis. The approach can be considered as an alternate strategy to disable certain transcription factor-mediated tumor growth.

\section{Acknowledgments}

This study was partially supported by the Translational Oncology Program Discovery Fund Award, Samuel Oschin Comprehensive Cancer Institute (SOCCI), Cedars-Sinai Medical Center (RM and XC),NIH R01CA151610 (XC) and BCRF (MIG). We would like to thank Dr. Akashi Otaki for the construct and preparations of recombinant RelA protein.

\section{Disclosure}

HK and RM are inventors in patent application related to the work and RM reports a US patent issued. The work has been licensed to Kairos Pharma, Ltd. The authors reported no other potential conflicts of interest for this work.

\section{References}

1. Keenan TE, Tolaney SM. Role of immunotherapy in triple-negative breast cancer. J Natl Compr Canc Netw. 2020;18(4):479-489. doi:10.6004/jnccn.2020.7554

2. Waks AG, Winer EP. Breast cancer treatment: a review. JAMA. 2019;321(3):288-300. doi:10.1001/jama.2018.19323

3. Cao Y, Karin M. NF-kappaB in mammary gland development and breast cancer. J Mammary Gland Biol Neoplasia. 2003;8(2):215-223. doi:10.1023/A:1025905008934

4. Heiser LM, Sadanandam A, Kuo WL, et al. Subtype and pathway specific responses to anticancer compounds in breast cancer. Proc Natl Acad Sci U S A. 2012;109(8):2724-2729. doi:10.1073/pnas.1018854108

5. Subbaramaiah K, Howe LR, Bhardwaj P, et al. Obesity is associated with inflammation and elevated aromatase expression in the mouse mammary gland. Cancer Prev Res. 2011;4(3):329-346. doi:10.1158/ 1940-6207.CAPR-10-0381

6. Egusquiaguirre SP, Yeh JE, Walker SR, Liu S, Frank DA. The STAT3 target gene TNFRSF1A modulates the NF-kappaB pathway in breast cancer cells. Neoplasia (New York, NY). 2018;20(5):489-498. doi:10.1016/j.neo.2018.03.004

7. Li HH, Zhu H, Liu LS, et al. Tumour necrosis factor-alpha gene polymorphism is associated with metastasis in patients with triple negative breast cancer. Sci Rep. 2015;5:10244. doi:10.1038/srep10244

8. Wee ZN, Yatim SM, Kohlbauer VK, et al. IRAK1 is a therapeutic target that drives breast cancer metastasis and resistance to paclitaxel. Nat Commun. 2015;6:8746. doi:10.1038/ncomms9746
9. Wang W, Nag SA, Zhang R. Targeting the NFкB signaling pathways for breast cancer prevention and therapy. Curr Med Chem. 2015;22 (2):264-289. doi:10.2174/0929867321666141106124315

10. Kanzaki H, Mukhopadhya NK, Cui X, Ramanujan VK, Murali R. Trastuzumab-resistant luminal $\mathrm{b}$ breast cancer cells show basal-like cell growth features through NF-kappaB-activation. Monoclon Antib Immunodiagn Immunother. 2016;35(1):1-11. doi:10.1089/mab.20 15.0056

11. Oliveras-Ferraros C, Vazquez-Martin A, Martin-Castillo B, et al. Pathway-focused proteomic signatures in HER2-overexpressing breast cancer with a basal-like phenotype: new insights into de novo resistance to trastuzumab (Herceptin). Int J Oncol. 2010;37 (3):669-678. doi:10.3892/ijo_00000716

12. Nakshatri H, Bhat-Nakshatri P, Martin DA, Goulet RJ Jr, Sledge GW Jr. Constitutive activation of NF-kappaB during progression of breast cancer to hormone-independent growth. Mol Cell Biol. 1997;17 (7):3629-3639. doi:10.1128/MCB.17.7.3629

13. Yamaguchi N, Ito $\mathrm{T}$, Azuma $\mathrm{S}$, et al. Constitutive activation of nuclear factor- $\mathrm{\kappa B}$ is preferentially involved in the proliferation of basal-like subtype breast cancer cell lines. Cancer Sci. 2009;100 (9):1668-1674. doi:10.1111/j.1349-7006.2009.01228.x

14. Baldwin AS Jr. The NF-kappa B and I kappa B proteins: new discoveries and insights. Annu Rev Immunol. 1996;14:649-683. doi:10.1146/annurev.immunol.14.1.649

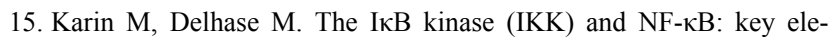
ments of proinflammatory signalling. Semin Immunol. 2000;12 (1):85-98. doi:10.1006/smim.2000.0210

16. Kim JY, Jung HH, Ahn S, et al. The relationship between nuclear factor (NF)-kappaB family gene expression and prognosis in triplenegative breast cancer (TNBC) patients receiving adjuvant doxorubicin treatment. Sci Rep. 2016;6:31804. doi:10.1038/srep31804

17. Umezawa K. Inhibition of tumor growth by NF-kappaB inhibitors. Cancer Sci. 2006;97(10):990-995. doi:10.1111/j.1349-7006.2006. 00285. $\mathrm{x}$

18. Lin Y, Ukaji T, Koide N, Umezawa K. Inhibition of late and early phases of cancer metastasis by the NF- $\mathrm{kB}$ inhibitor DHMEQ derived from microbial bioactive metabolite epoxyquinomicin: a review. Int $J$ Mol Sci. 2018;19(3):729. doi:10.3390/ijms19030729

19. Netanely D, Avraham A, Ben-Baruch A, Evron E, Shamir R. Expression and methylation patterns partition luminal-A breast tumors into distinct prognostic subgroups. Breast Cancer Res. 2016;18(1):74. doi:10.1186/s13058-016-0724-2

20. Love MI, Huber W, Anders S. Moderated estimation of fold change and dispersion for RNA-seq data with DESeq2. Genome Biol. 2014;15(12):550. doi:10.1186/s13059-014-0550-8

21. Benjamini Y, Hochberg Y. Controlling the false discovery rate: a practical and powerful approach to multiple testing. $J R$ Stat Soc Series B Stat Methodol. 1995;57:289-300.

22. Murali R, Cheng X, Berezov A, et al. Disabling TNF receptor signaling by induced conformational perturbation of tryptophan107. Proc Natl Acad Sci U S A. 2005;102(31):10970-10975. doi:10.1073/pnas.0504301102

23. Berezov A, Cai Z, Freudenberg JA, et al. Disabling the mitotic spindle and tumor growth by targeting a cavity-induced allosteric site of survivin. Oncogene. 2012;31(15):1938-1948. doi:10.1038/ onc. 2011.377

24. Chen Y-Q, Ghosh S, Ghosh G. A novel DNA recognition mode by the NF-кB p65 homodimer. Nat Struct Biol. 1998;5(1):67-73. doi:10.1038/nsb0198-67

25. Jacobs MD, Harrison SC. Structure of an I $\mathrm{B} \alpha / \mathrm{NF}-\kappa \mathrm{B}$ complex. Cell. 1998;95(6):749-758. doi:10.1016/S0092-8674(00)81698-0

26. Chen FE, Huang D-B, Chen Y-Q, Ghosh G. Crystal structure of p50/ p65 heterodimer of transcription factor NF- $\mathrm{kB}$ bound to DNA. Nature. 1998;391(6665):410-413. doi:10.1038/34956

27. Morin A, Eisenbraun B, Key J, et al. Collaboration gets the most out of software. eLife. 2013;2:e01456. doi:10.7554/eLife.01456 
28. Pratt MA, Tibbo E, Robertson SJ, et al. The canonical NF-kappaB pathway is required for formation of luminal mammary neoplasias and is activated in the mammary progenitor population. Oncogene. 2009;28(30):2710-2722. doi:10.1038/onc.2009.131

29. Shostak K, Chariot A. NF-kappaB, stem cells and breast cancer: the links get stronger. Breast Cancer Res. 2011;13(4):214. doi:10.1186/ bcr2886

30. Jones RL, Rojo F, A'Hern R, et al. Nuclear NF-kappaB/p65 expression and response to neoadjuvant chemotherapy in breast cancer. $J$ Clin Pathol. 2011;64(2):130-135. doi:10.1136/jcp.2010.082966

31. Wang L, Kang F, Li J, Zhang J, Shan B. Overexpression of p65 attenuates celecoxib-induced cell death in MDA-MB-231 human breast cancer cell line. Cancer Cell Int. 2013;13(1):14. doi:10.1186/ 1475-2867-13-14

32. Jubb H, Higueruelo AP, Winter A, Blundell TL. Structural biology and drug discovery for protein-protein interactions. Trends Pharmacol Sci. 2012;33(5):241-248. doi:10.1016/j.tips.2012.03.006

33. Hartman ZC, Poage GM, den Hollander P, et al. Growth of triplenegative breast cancer cells relies upon coordinate autocrine expression of the proinflammatory cytokines IL-6 and IL-8. Cancer Res. 2013;73(11):3470-3480. doi:10.1158/0008-5472.CAN-12-4524-T

34. Lee ST, Li Z, Wu Z, et al. Context-specific regulation of NF-kappaB target gene expression by EZH2 in breast cancers. Mol Cell. 2011;43 (5):798-810. doi:10.1016/j.molcel.2011.08.011

35. Li S, Kendall SE, Raices R, et al. TWIST1 associates with NFkappaB subunit RELA via carboxyl-terminal WR domain to promote cell autonomous invasion through IL8 production. BMC Biol. 2012;10:73. doi:10.1186/1741-7007-10-73

36. Park B-W, Zhang H-T, Wu C, et al. Rationally designed anti-HER2/ neu peptide mimetic disables P185HER2/neu tyrosine kinases in vitro and in vivo. Nat Biotechnol. 2000;18(2):194-198. doi:10.1038/72651

37. Gupta SC, Sundaram C, Reuter S, Aggarwal BB. Inhibiting NF- $\kappa B$ activation by small molecules as a therapeutic strategy. Biochim Biophys Acta. 2010;1799(10-12):775-787. doi:10.1016/j.bbagrm.20 10.05.004
38. Baud V, Karin M. Is NF-kappaB a good target for cancer therapy? Hopes and pitfalls. Nat Rev Drug Discov. 2009;8(1):33-40. doi:10.1038/nrd2781

39. Umezawa K. Possible role of peritoneal NF- $\kappa B$ in peripheral inflammation and cancer: lessons from the inhibitor DHMEQ. Biomed Pharmacother. 2011;65(4):252-259. doi:10.1016/j.biopha.2011.02. 003

40. Murali R, Greene MI, Inventors; (The Trustees of the University of Pennsylvania, USA). assignee. Cavity-induced allosteric modification of intermolecular interactions and methods of identifying compounds that effect them. US patent 99-US15062 2000001349. 19990701. 2000.

41. Chen X, Kandasamy K, Srivastava RK. Differential roles of RelA (p65) and c-Rel subunits of nuclear factor kappa B in tumor necrosis factor-related apoptosis-inducing ligand signaling. Cancer Res. 2003;63(5):1059-1066.

42. Jacque E, Billot K, Authier H, Bordereaux D, Baud V. RelB inhibits cell proliferation and tumor growth through p53 transcriptional activation. Oncogene. 2013;32(21):2661-2669. doi:10.1038/onc.2012. 282

43. Ding H, Gangalum PR, Galstyan A, et al. HER2-positive breast cancer targeting and treatment by a peptide-conjugated mini nanodrug. Nanomedicine. 2017;13(2):631-639. doi:10.1016/j.nano.2016. 07.013

44. Levine DH, Ghoroghchian PP, Freudenberg J, et al. Polymersomes: a new multi-functional tool for cancer diagnosis and therapy. Methods. 2008;46(1):25-32. doi:10.1016/j.ymeth.2008.05.006

45. Guillemard V, Nedev HN, Berezov A, Murali R, Saragovi HU. HER2-mediated internalization of a targeted prodrug cytotoxic conjugate is dependent on the valency of the targeting ligand. DNA Cell Biol. 2005;24(6):350-358. doi:10.1089/dna.2005.24.351

46. DeLano WL The PyMOL Molecular Graphics System. Palo Alto, CA, USA: DeLando Scientific LLC; 2007. Available from: http:// www.pymol.org. Accessed June 17, 2021.
Breast Cancer: Targets and Therapy

\section{Publish your work in this journal}

Breast Cancer - Targets and Therapy is an international, peer-reviewed open access journal focusing on breast cancer research, identification of therapeutic targets and the optimal use of preventative and integrated treatment interventions to achieve improved outcomes, enhanced survival and quality of life for the cancer patient.
The manuscript management system is completely online and includes a very quick and fair peer-review system, which is all easy to use. Visit http://www.dovepress.com/testimonials.php to read real quotes from published authors. 